\begin{tabular}{|c|c|c|c|c|c|c|}
\hline \multirow{4}{*}{ Impact Factor: } & ISRA (India) & $=3.117$ & SIS (USA) & $=0.912$ & ICV (Poland) & $=6.630$ \\
\hline & ISI (Dubai, UAE & $=0.829$ & РИНЦ (Russia) & $=0.156$ & PIF (India) & $=1.940$ \\
\hline & GIF (Australia) & $=0.564$ & ESJI (KZ) & $=8.716$ & IBI (India) & $=4.260$ \\
\hline & JIF & $=1.500$ & SJIF (Morocco) & $=5.667$ & OAJI (USA) & $=0.350$ \\
\hline
\end{tabular}

\section{SOI: $1.1 /$ TAS $\quad$ DOI: $10.15863 /$ TAS International Scientific Journal Theoretical \& Applied Science}

p-ISSN: 2308-4944 (print) e-ISSN: 2409-0085 (online)

Year: $2019 \quad$ Issue: $05 \quad$ Volume: 73

Published: $10.05 .2019 \quad \underline{\text { http://T-Science.org }}$

SECTION 2. Applied mathematics.

Mathematical modeling.
QR - Issue

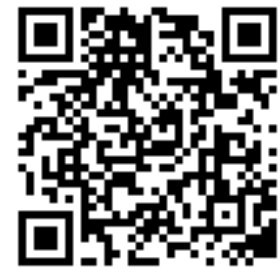

Meruyet Murat master student,

Taraz State University named after M.Kh. Dulati

Alexandr Shevtsov candidate of technical sciences, member of PILA (USA), department of «Mathematics», deputy director on Science of faculty of Information technologies, automation and telecommunications, Taraz State University named after M.Kh. Dulati Shev_AlexXXXX@mail.ru

\title{
LIBRARY FOR SOME NUMERICAL OPTIMIZATION ALGORITHMS
}

Abstract: In the article the library for some algorithms of realization of optimization problems in the system of computer algebra Maple is developed.

Key words: Maple, equation, library.

Language: English

Citation: Murat, M., \& Shevtsov, A. (2019). Library for some numerical optimization algorithms. ISJ Theoretical \& Applied Science, 05 (73), 254-265.

Soi: http://s-o-i.org/1.1/TAS-05-73-36 Doi: crossef https://dx.doi.org/10.15863/TAS.2019.05.73.36

\section{Introduction}

Some numerical methods for solving the optimization problem are based on the exact or approximate calculation of its characteristics cannot be solved by the standard library Maple [1-10]. Therefore, by collecting separate algorithms for solving the optimization problem in a separate library, we can contribute to the solution of this problem [911].

Let us consider the process of developing a library to implement an optimization algorithm in a Maple computer algebra system.

\section{Materials and Methods}

The development of a library of Maple NumOpt_MuratM.MapleLib for solving some numerical optimization problems

Library development NumOpt_MuratM. MapleLib implementing the chord method

Numopt library code: 


\begin{tabular}{|c|c|c|c|c|c|c|}
\hline \multirow{4}{*}{ Impact Factor: } & ISRA (India) & $=3.117$ & SIS (USA) & $=0.912$ & ICV (Poland) & $=6.630$ \\
\hline & ISI (Dubai, UAE & $=0.829$ & РИНЦ (Russia & $=0.156$ & PIF (India) & $=1.940$ \\
\hline & GIF (Australia) & $=0.564$ & ESJI (KZ) & $=8.716$ & IBI (India) & $=4.260$ \\
\hline & JIF & $=1.500$ & SJIF (Morocce & $=5.667$ & OAJI (USA) & $=0.350$ \\
\hline
\end{tabular}

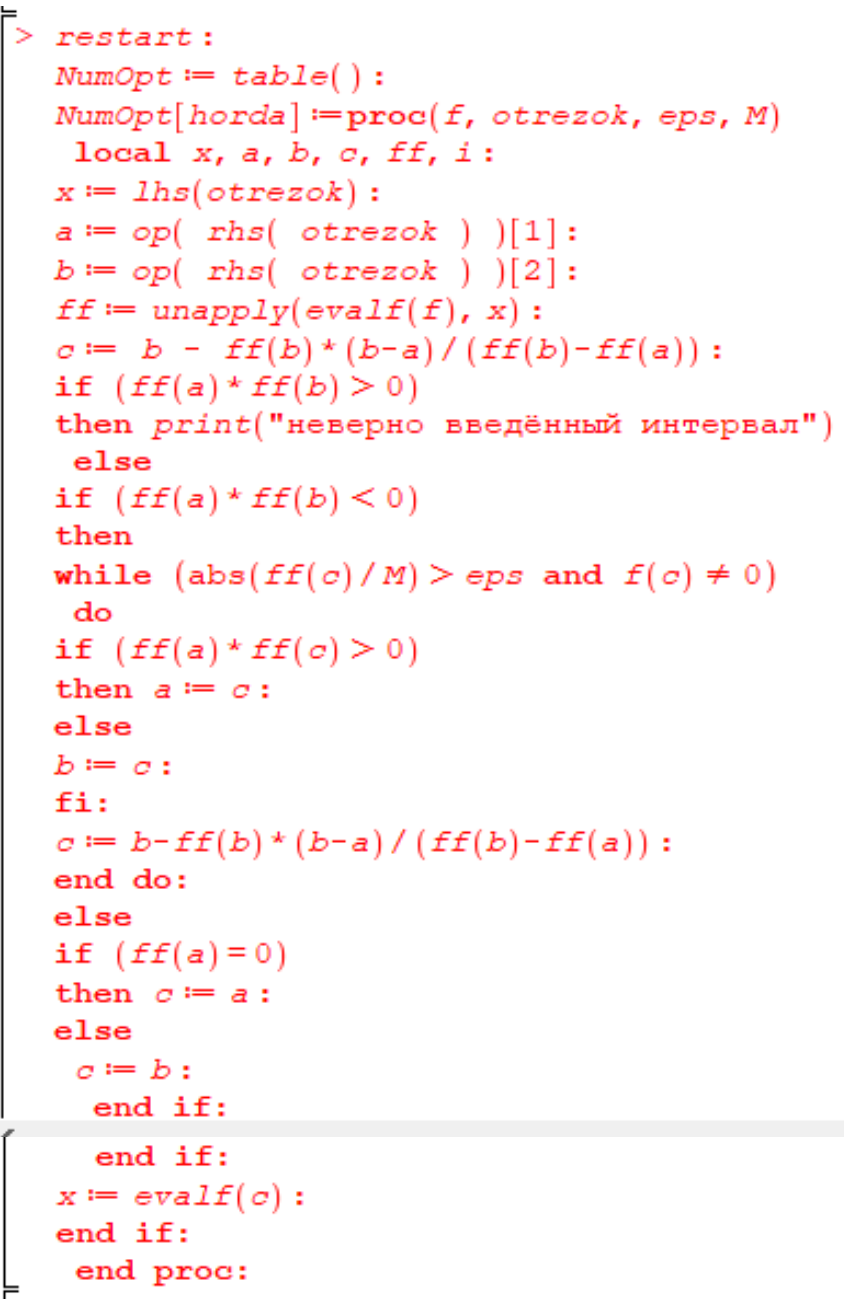

Connect the library

$>\operatorname{read}\left({ }^{\prime} D \cdot /\right.$ NumOpt_MuratMMapleLib');

with $(\mathrm{NumOpt})$;

[bisection, dixotomi, gold, horda, newton, newton_mod] chords:

Code using the library NumOpt for method

$$
\begin{aligned}
& \mathrm{f}:=\mathrm{x} \rightarrow \mathrm{x}-5 \cdot \sin \left(\frac{1}{(x-1)^{2}+1}\right) ; f 1:=x-5 \cdot \sin \left(\frac{1}{(x-1)^{2}+1}\right) ; \\
& \begin{aligned}
a:=0 ; b:=3.5 ; \text { eps }:=10^{-3} ; & \\
& f:=x \mapsto x-5 \sin \left(\frac{1}{(x-1)^{2}+1}\right) \\
& f 1:=x-5 \sin \left(\frac{1}{(x-1)^{2}+1}\right) \\
& a:=0 \\
& b:=3.5 \\
& \text { eps }:=\frac{1}{1000}
\end{aligned}
\end{aligned}
$$

graph $:=\operatorname{plot}(f 1, x=a . . b$, color $=$ red, thickness $=3) ;$ 


\begin{tabular}{llllll} 
& ISRA (India) $=\mathbf{3 . 1 1 7}$ & SIS (USA) $=\mathbf{0 . 9 1 2}$ & ICV (Poland) & $\mathbf{= 6 . 6 3 0}$ \\
Impact Factor: & ISI (Dubai, UAE) $=\mathbf{0 . 8 2 9}$ & PUHЦ (Russia) $=\mathbf{0 . 1 5 6}$ & PIF (India) & $=\mathbf{1 . 9 4 0}$ \\
& GIF (Australia) $=\mathbf{0 . 5 6 4}$ & ESJI (KZ) $=\mathbf{8 . 7 1 6}$ & IBI (India) & $=\mathbf{4 . 2 6 0}$ \\
& JIF & $\mathbf{1 . 5 0 0}$ & SJIF (Morocco) $=\mathbf{5 . 6 6 7}$ & OAJI (USA) & $\mathbf{0 . 3 5 0}$ \\
\hline
\end{tabular}

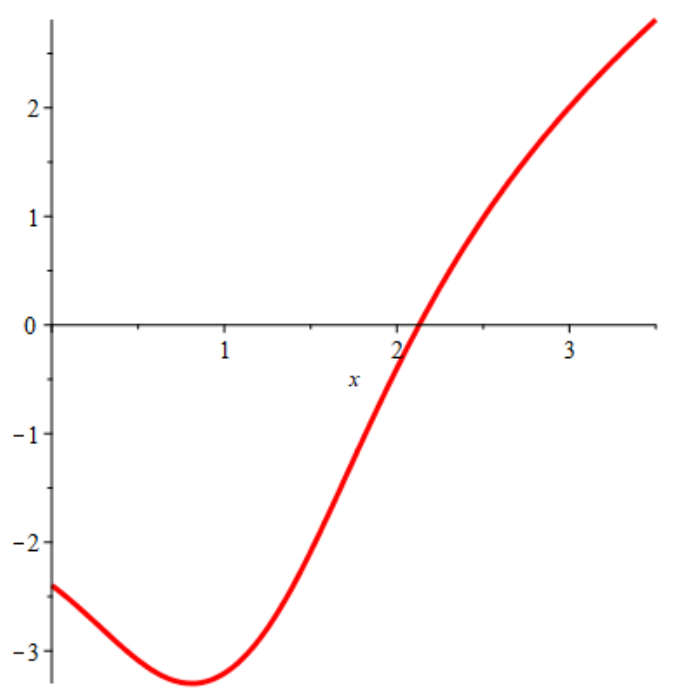

$f 2:=\operatorname{diff}(f 1, x)$;

$x_{-} x о p \partial a:=\operatorname{horda}(f 2, x=a . . b$, eps, 1$)$;

$y_{-}$хорда $a:=f\left(x_{-}\right.$хорда $)$;

with(plots) :

$p:=$ pointplot $\left(\left[x \_x o p \partial a, y \_x o p \partial a\right]\right.$, color $=$ blue $):$

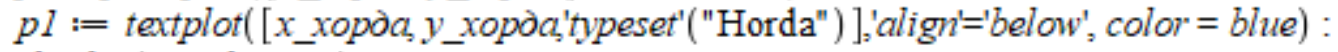

display (graph, pl, p);

$$
\begin{aligned}
& f 2:=1+\frac{5(2 x-2) \cos \left(\frac{1}{(x-1)^{2}+1}\right)}{\left((x-1)^{2}+1\right)^{2}} \\
& x \_ \text {хорда } a:=0.8113132771 \\
& \text { y_хорда }:=-3.300699391
\end{aligned}
$$

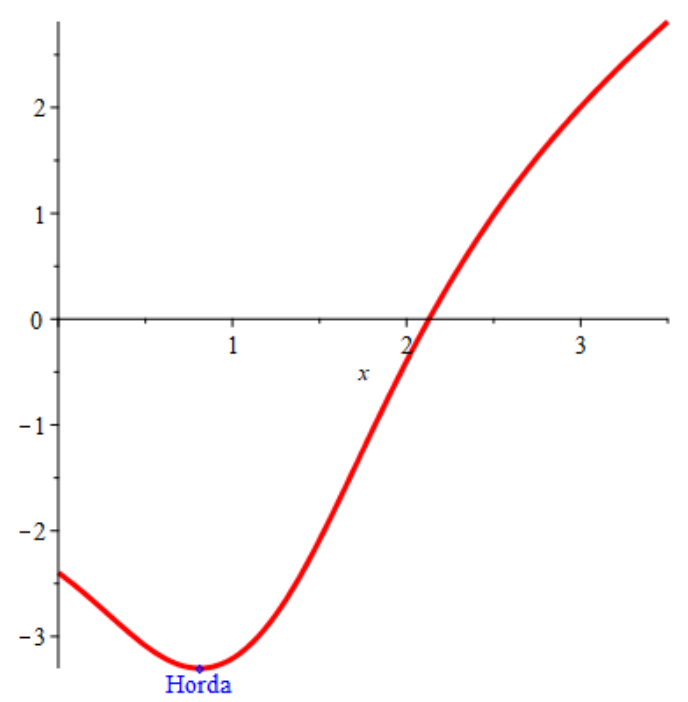

Library

NumOpt_MuratM.MapleLib

development

Golden section method

Numopt library code: 


\begin{tabular}{|c|c|c|c|c|c|c|}
\hline \multirow{4}{*}{ Impact Factor: } & ISRA (India) & $=3.117$ & SIS (USA) & $=0.912$ & ICV (Poland) & $=6.630$ \\
\hline & ISI (Dubai, UAE & $=0.829$ & РИНЦ (Russia) & $=0.156$ & PIF (India) & $=1.940$ \\
\hline & GIF (Australia) & $=0.564$ & ESJI (KZ) & $=8.716$ & IBI (India) & $=4.260$ \\
\hline & JIF & $=1.500$ & SJIF (Morocco) & $=5.667$ & OAJI (USA) & $=0.350$ \\
\hline
\end{tabular}

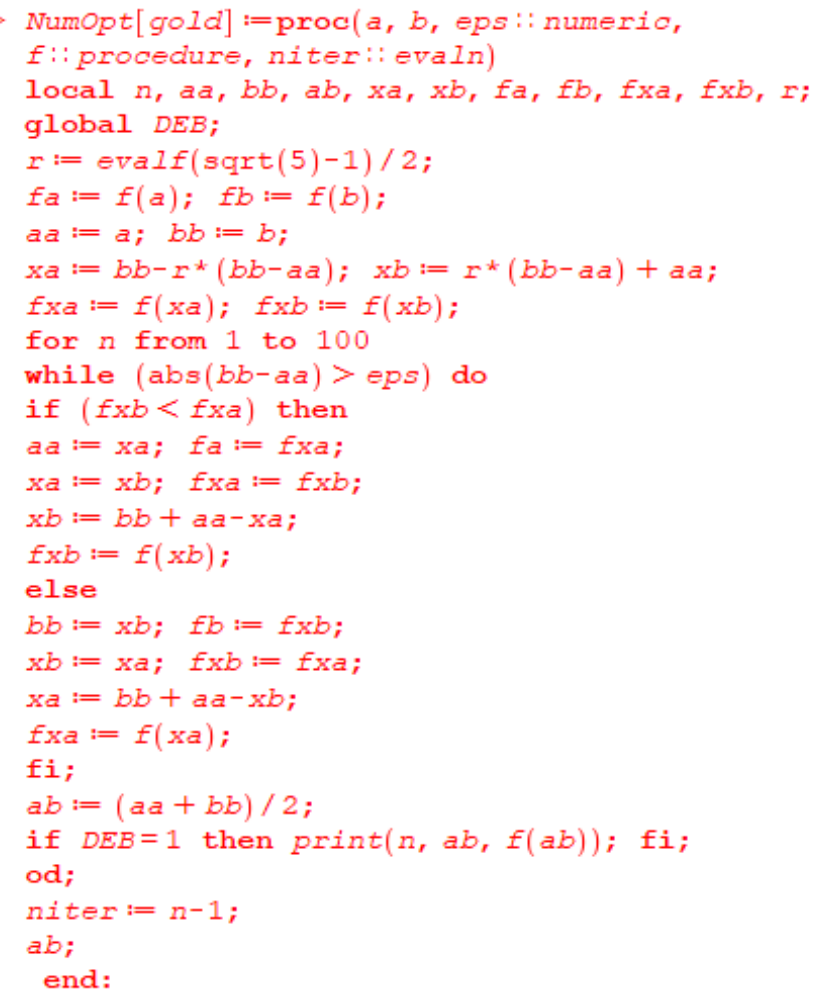

Code using the library NumOpt for the method of the Golden section:

$x_{-}$золотоесечение $:=\operatorname{gold}(\operatorname{eval} f(a)$, evalf $(b), \operatorname{eps}, f, n)$;

$y$ золотоесечение $:=f\left(x \_\right.$золотоесечение $)$;

with(plots) :

$s:=$ pointplot $\left(\left[x_{-}\right.\right.$золотоесечение, $y_{-}$золотоесечение $]$, color $=$blue $)$;

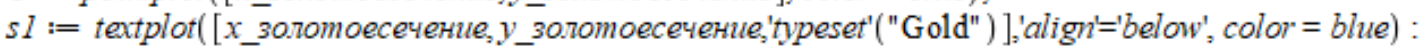
$\operatorname{display}($ graph, s, sl);

$$
\begin{aligned}
& x \text { _золотоесечение }:=0.8113338800 \\
& y \_ \text {золотоесечение }:=-3.300699407
\end{aligned}
$$

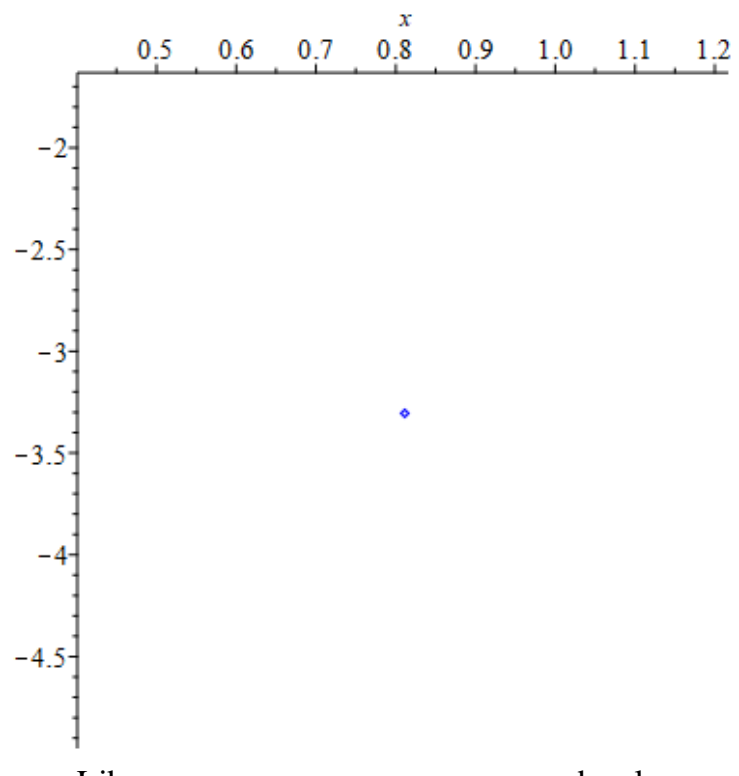

Library NumOpt Mu of dichotomy

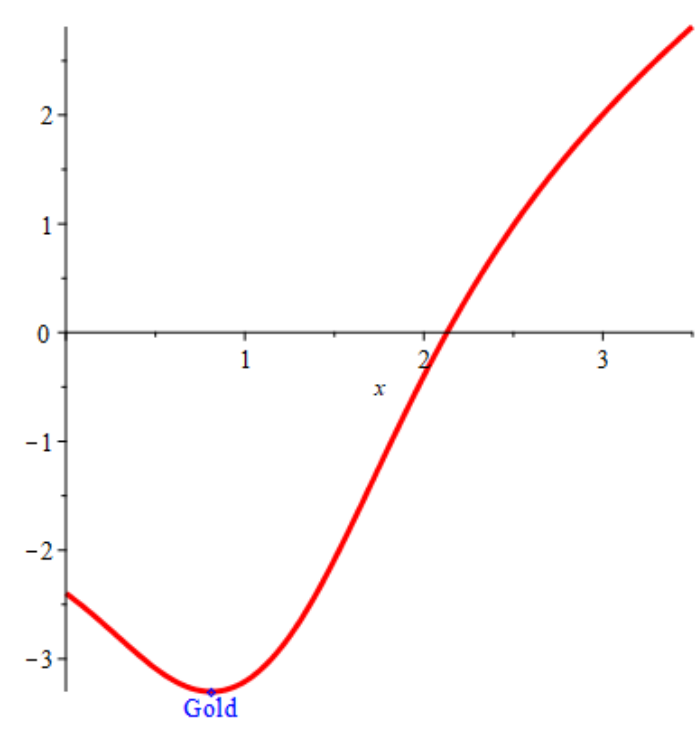

Numopt library code: 


\begin{tabular}{|c|c|c|c|c|c|c|}
\hline \multirow{4}{*}{ Impact Factor: } & ISRA (India) & $=3.117$ & SIS (USA) & $=0.912$ & ICV (Poland) & $=6.630$ \\
\hline & ISI (Dubai, UAE & $=0.829$ & РИНЦ (Russia) & $=0.156$ & PIF (India) & $=1.940$ \\
\hline & GIF (Australia) & $=0.564$ & ESJI (KZ) & $=8.716$ & IBI (India) & $=4.260$ \\
\hline & JIF & $=1.500$ & SJIF (Morocco & $=5.667$ & OAJI (USA) & $=0.350$ \\
\hline
\end{tabular}

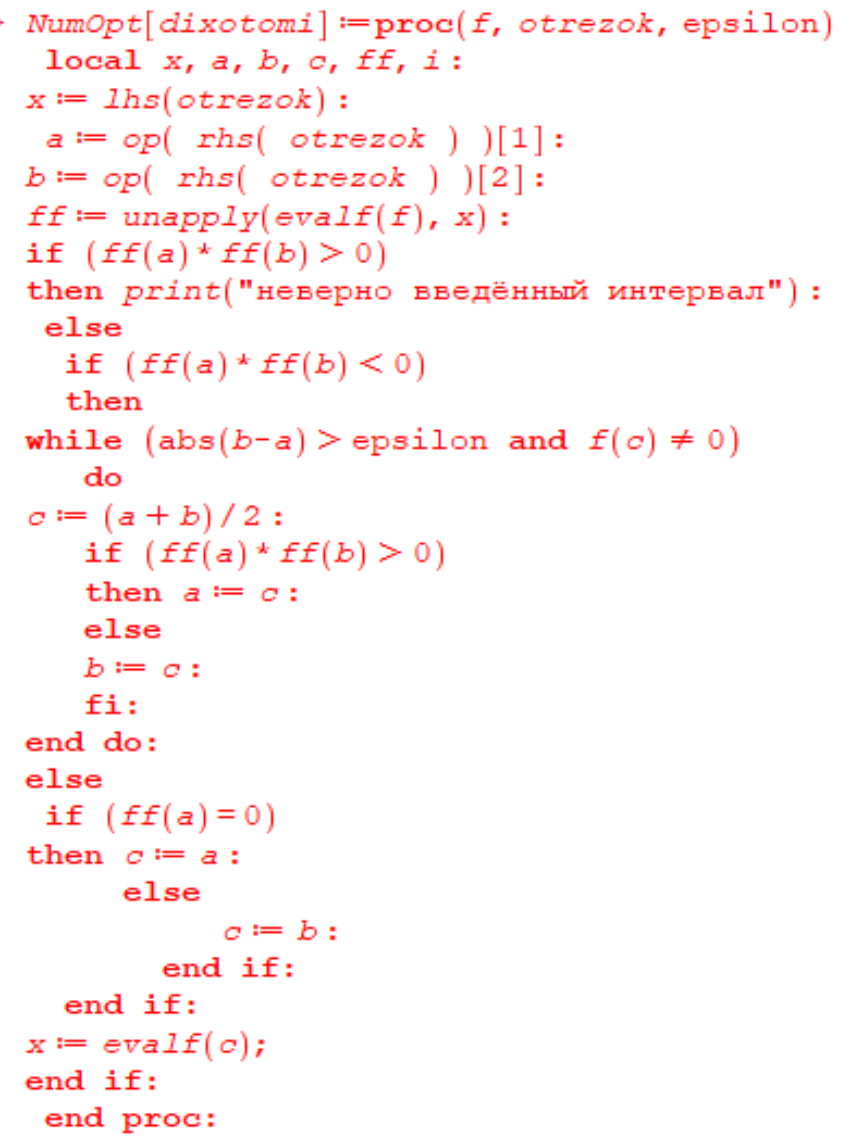

Code using the library NumOpt for the method of dichotomy:

$x \_$дxхотоми $:=\operatorname{dixotomi}(f 1, x=a . . b$, eps $)$;

$y_{-}$дихотоми $:=f\left(x_{-}\right.$дихотоми $)$;

with $($ plots $): e:=$ pointplot $\left(\left[x_{-}\right.\right.$дuxomomu,y_duxomomu $]$, color $=$blue $)$:

$e 1:=$ textplot $\left(\left[x_{-}\right.\right.$drxomomu, $y_{-}$duxomomu'typeset' $($"Dixotomi" $\left.)\right]$, 'align'='below', color $=$blue $)$:

$\operatorname{display}($ graph, $e, \overline{e l})$;

$$
\begin{gathered}
x \_ \text {_ихотоми }:=1.749145508 \\
y \_ \text {дихотоми }:=-1.238937316
\end{gathered}
$$

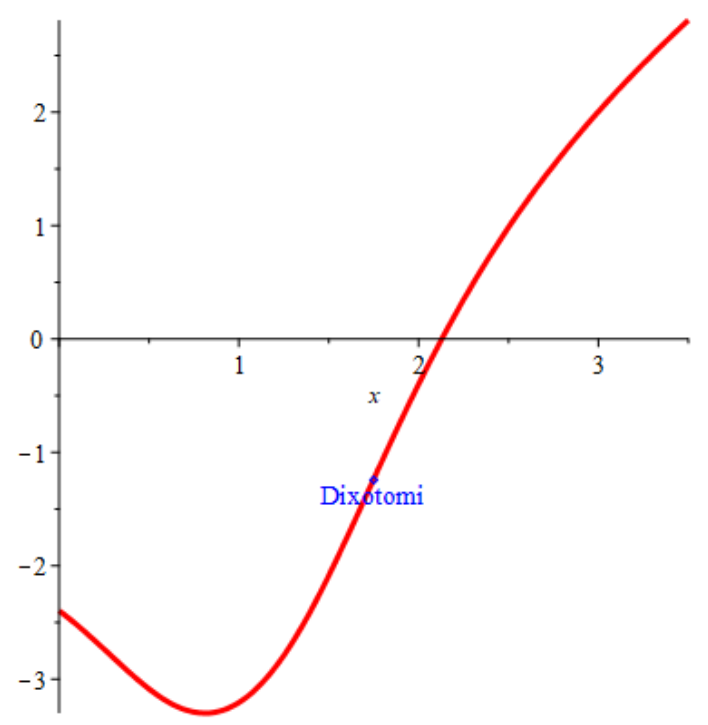




\begin{tabular}{|c|c|c|c|c|c|c|}
\hline \multirow{4}{*}{ Impact Factor: } & ISRA (India) & $=3.117$ & SIS (USA) & $=0.912$ & ICV (Poland) & $=6.630$ \\
\hline & ISI (Dubai, UAE & $=0.829$ & РИНЦ (Russia & $=0.156$ & PIF (India) & $=1.940$ \\
\hline & GIF (Australia) & $=0.564$ & ESJI (KZ) & $=8.716$ & IBI (India) & $=4.260$ \\
\hline & JIF & $=1.500$ & SJIF (Morocce & $=5.667$ & OAJI (USA) & $=0.350$ \\
\hline
\end{tabular}

Library

development

NumOpt_MuratM.MapleLib implementing Newton's method

Numopt library code

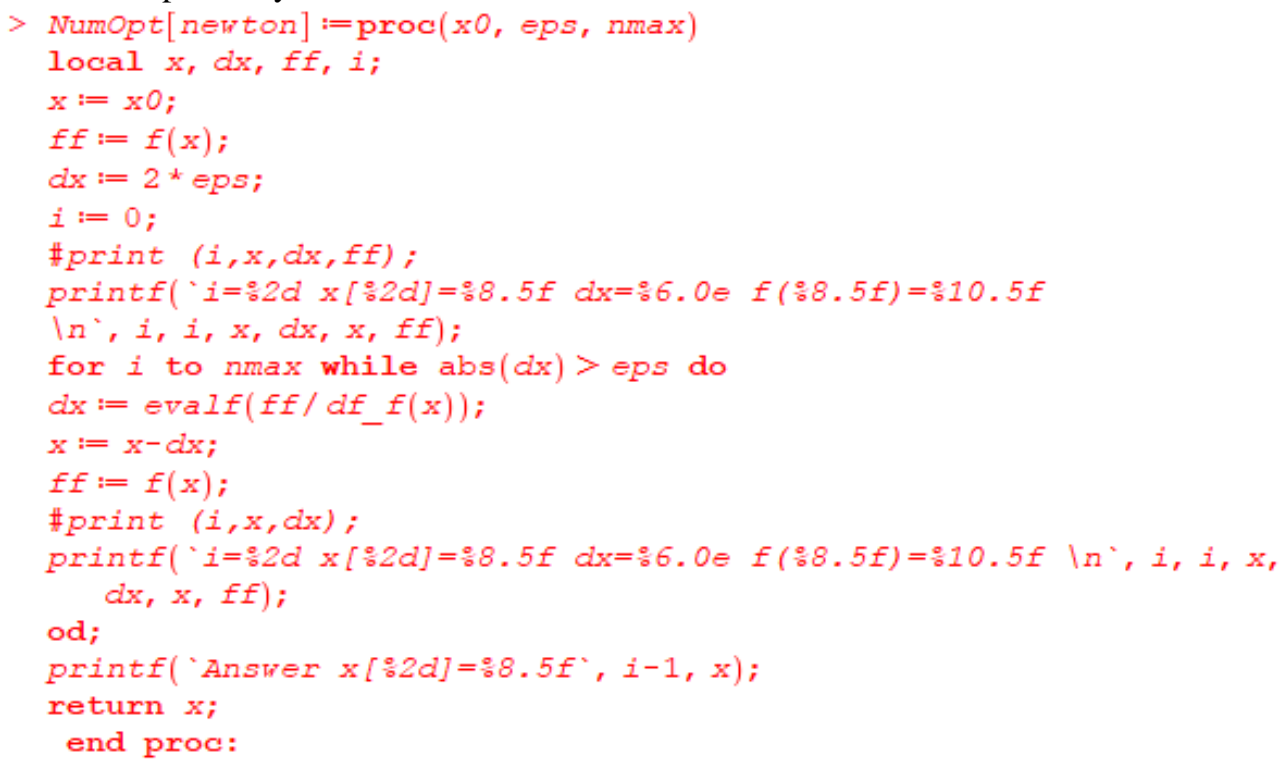

Code for using the NumOpt library for the

Newton method

$f a:=f(a)$;

$d f:=\operatorname{diff}(f 1, x \$ 1)$;

$\operatorname{subs}(x=a, d f)$

dff $f:=\operatorname{unapply}(d f ; x)$;

evalf $($ df $f(a))$;

$x_{-}$нъютон $:=\operatorname{newton}(0.5$, eps, $n)$;

$y_{-}$ньютон $:=f\left(x_{-}\right.$нъютон $)$;

with $($ plots $): r:=$ pointplot $\left(\left[x_{-}\right.\right.$ньютон, $y_{-}$ньютон $]$, color $=$blue $)$:

$r l:=$ textplot $\left(\left[x_{-}\right.\right.$ньютон, $y_{-}$ньютонон'typeset' ("Newton" $\left.)\right]$'align'='below', color = blue $)$:

display $($ graph $, r, r l)$;

$$
\begin{gathered}
f a:=-5 \sin \left(\frac{1}{2}\right) \\
d f:=1+\frac{5(2 x-2) \cos \left(\frac{1}{(x-1)^{2}+1}\right)}{\left((x-1)^{2}+1\right)^{2}} \\
\text { If } f:=x \mapsto 1+\frac{5 \cos \left(\frac{1}{2}\right)}{2} \\
-1.193956405
\end{gathered}
$$




\begin{tabular}{|c|c|c|c|c|c|c|}
\hline \multirow{4}{*}{ Impact Factor: } & ISRA (India) & $=3.117$ & SIS (USA) & $=0.912$ & ICV (Poland) & $=6.630$ \\
\hline & ISI (Dubai, UAE & $=\mathbf{0 . 8 2 9}$ & РИНЦ (Russia) & $=0.156$ & PIF (India) & $=1.940$ \\
\hline & GIF (Australia) & $=0.564$ & ESJI (KZ) & $=8.716$ & IBI (India) & $=4.260$ \\
\hline & JIF & $=1.500$ & SJIF (Morocco & $=5.667$ & OAJI (USA) & $=0.350$ \\
\hline
\end{tabular}

\section{$-1.193956405$}
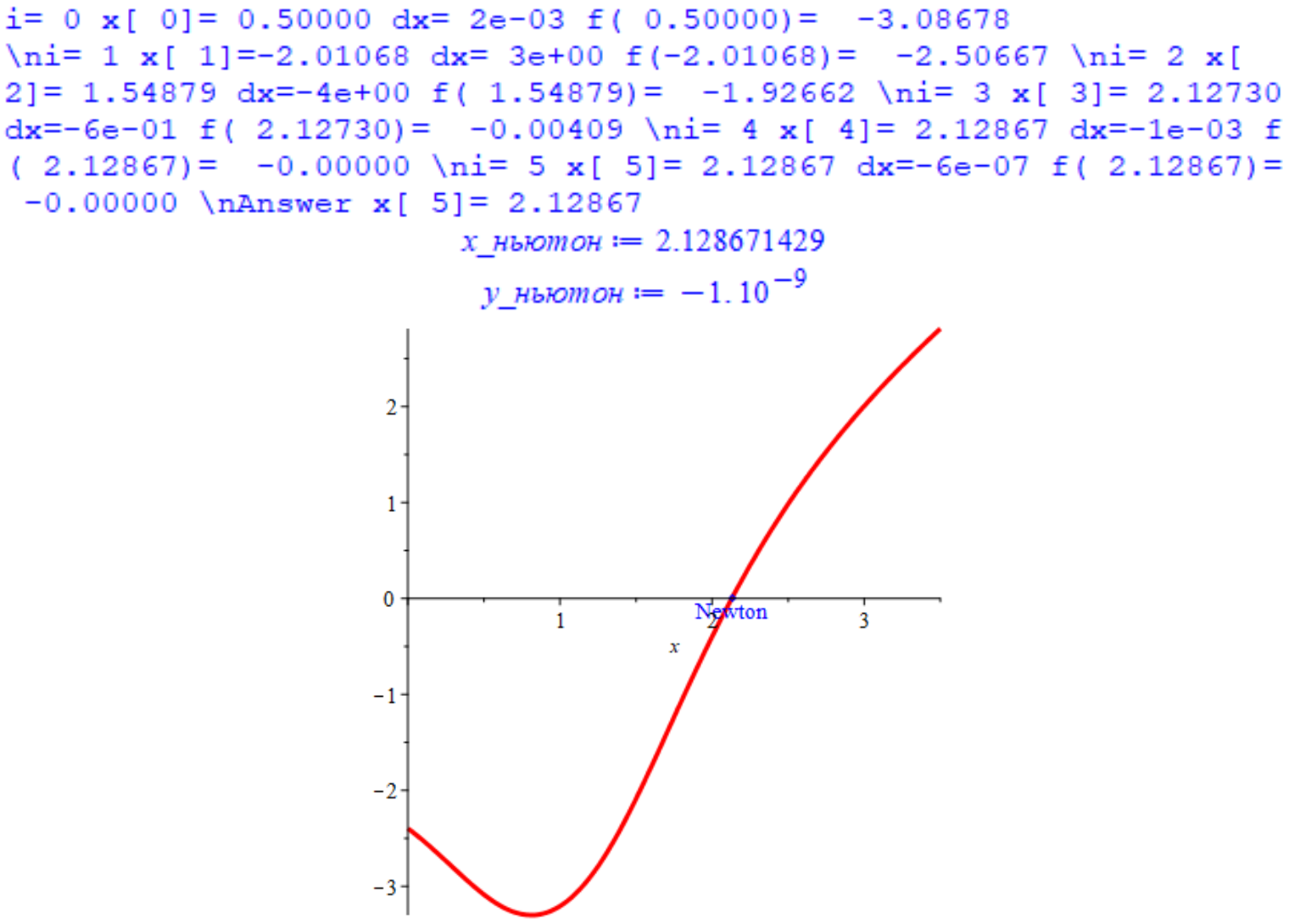

Library

development

NumOpt_MuratM.MapleLib implementing the modified Newton method

Numopt library code:

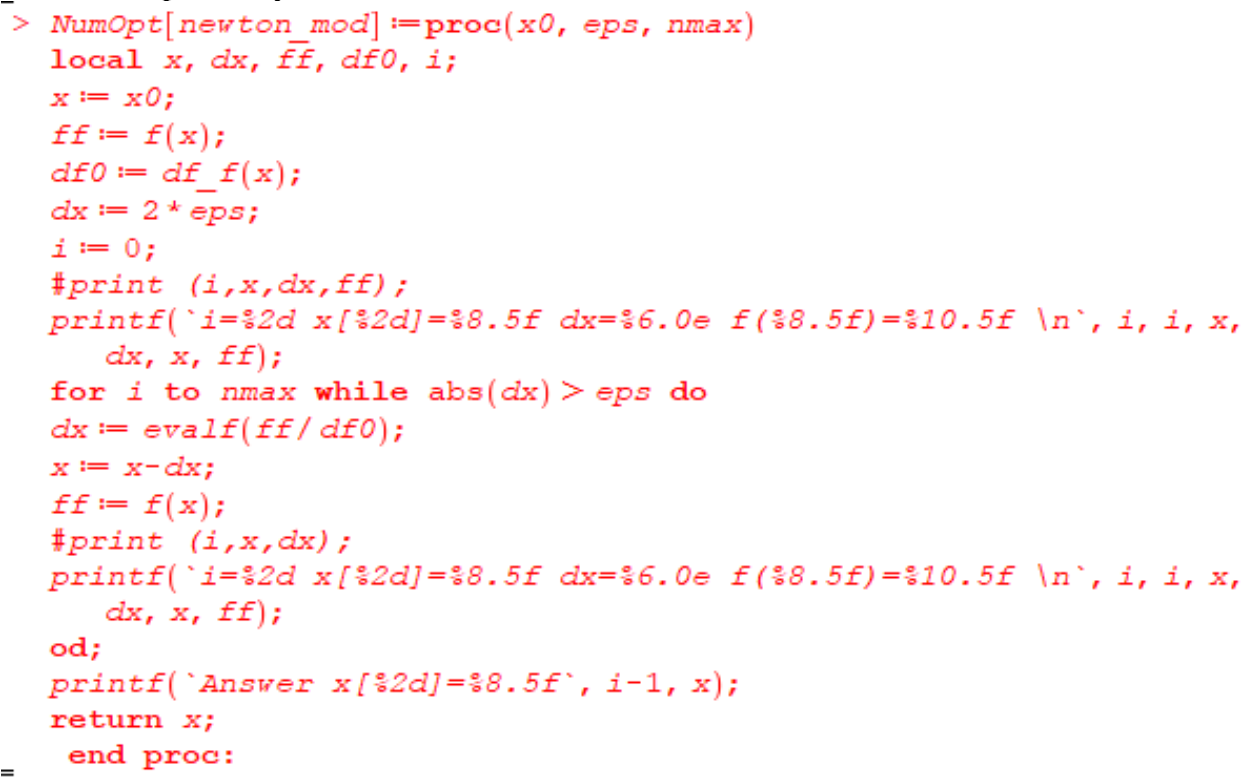

Library development NumOpt_MuratM.MapleLib implementing the bisection method

Numopt library code: 


\begin{tabular}{llllll} 
& ISRA (India) $=\mathbf{3 . 1 1 7}$ & SIS (USA) & $=\mathbf{0 . 9 1 2}$ & ICV (Poland) & $=\mathbf{6 . 6 3 0}$ \\
Impact Factor: & ISI (Dubai, UAE) $=\mathbf{0 . 8 2 9}$ & PUHL (Russia) $=\mathbf{0 . 1 5 6}$ & PIF (India) & $=\mathbf{1 . 9 4 0}$ \\
& GIF (Australia) $=\mathbf{0 . 5 6 4}$ & ESJI (KZ) & $=\mathbf{8 . 7 1 6}$ & IBI (India) & $=\mathbf{4 . 2 6 0}$ \\
& JIF & $\mathbf{1 . 5 0 0}$ & SJIF (Morocco) $=\mathbf{5 . 6 6 7}$ & OAJI (USA) & $\mathbf{0 . 3 5 0}$ \\
\hline
\end{tabular}

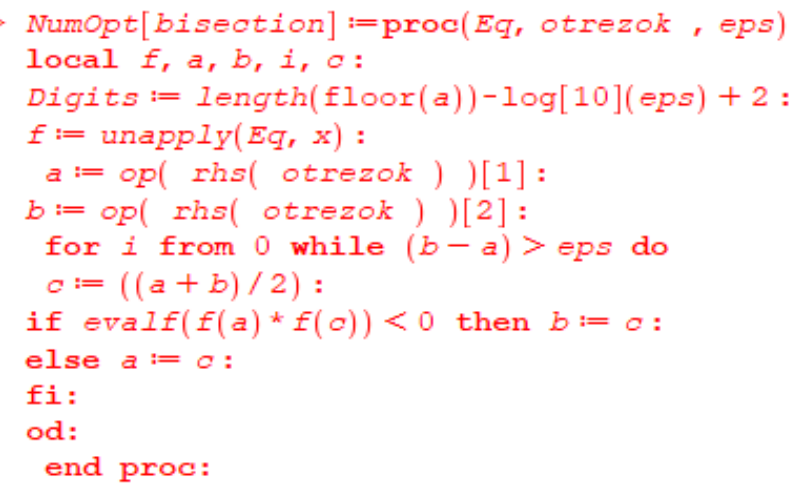
disk:

Next, save the created library to the computer save(NumOpt, 'D:/ NumOpt_MuratM.MapleLib");

Code for using the NumOpt library for the bisection method:

$E q:=\operatorname{diff}(f 1, x)$;

$x$ бucexunu := bisection $(E q, x=\operatorname{evalf}(a)$.evalf $(b)$, eps $)$;

$\bar{y}$ бисекиия : $=f\left(x_{-}\right.$бисекиия $)$;

with(plots) :

$m:=$ pointplot $\left(\left[x \_\right.\right.$бисекиия, $y \_$бисекиия $]$, color $=$blue $)$:

$m 1:=\operatorname{textplot}\left(\left[x \_\right.\right.$бисекиии, y_бисекиия,'typeset' $($"Bisekcia" $\left.)\right]$,'align'='below', color = blue $)$: $\operatorname{display}($ graph, $m, \mathrm{ml})$;

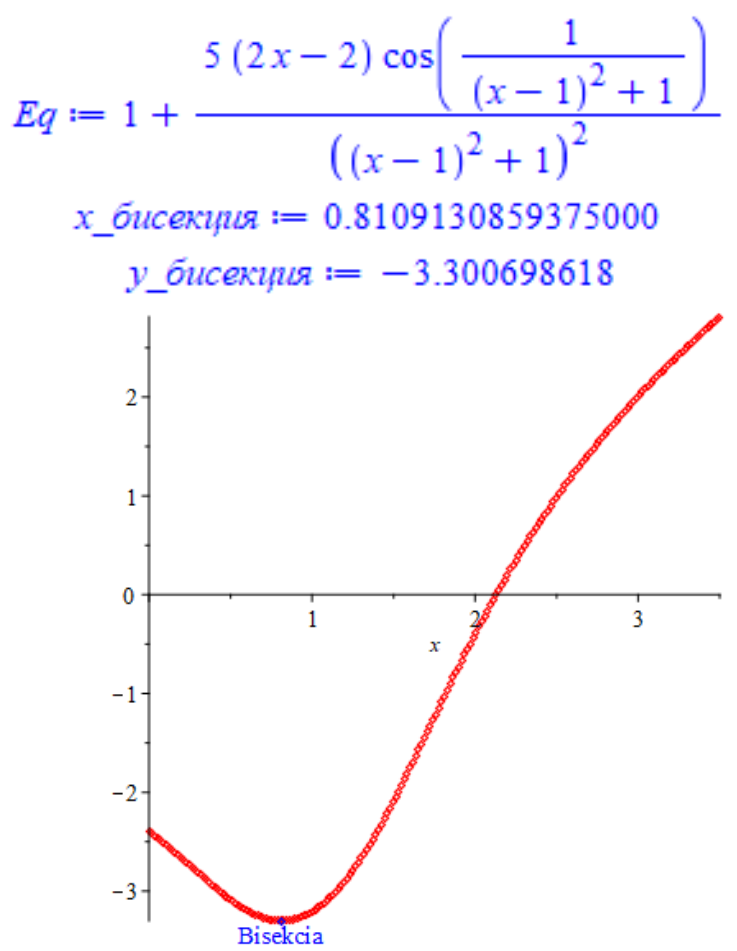

Library

development

NumOpt_MuratM.MapleLib implementing the search for a local minimum by the method of half division 


\begin{tabular}{llllll} 
& ISRA (India) $=\mathbf{3 . 1 1 7}$ & SIS (USA) & $=\mathbf{0 . 9 1 2}$ & ICV (Poland) & $=\mathbf{6 . 6 3 0}$ \\
Impact Factor: & ISI (Dubai, UAE) $=\mathbf{0 . 8 2 9}$ & PUHL (Russia) $=\mathbf{0 . 1 5 6}$ & PIF (India) & $=\mathbf{1 . 9 4 0}$ \\
& GIF (Australia) $=\mathbf{0 . 5 6 4}$ & ESJI (KZ) & $=\mathbf{8 . 7 1 6}$ & IBI (India) & $=\mathbf{4 . 2 6 0}$ \\
& JIF & $\mathbf{1 . 5 0 0}$ & SJIF (Morocco) $=\mathbf{5 . 6 6 7}$ & OAJI (USA) & $\mathbf{0 . 3 5 0}$ \\
\hline
\end{tabular}

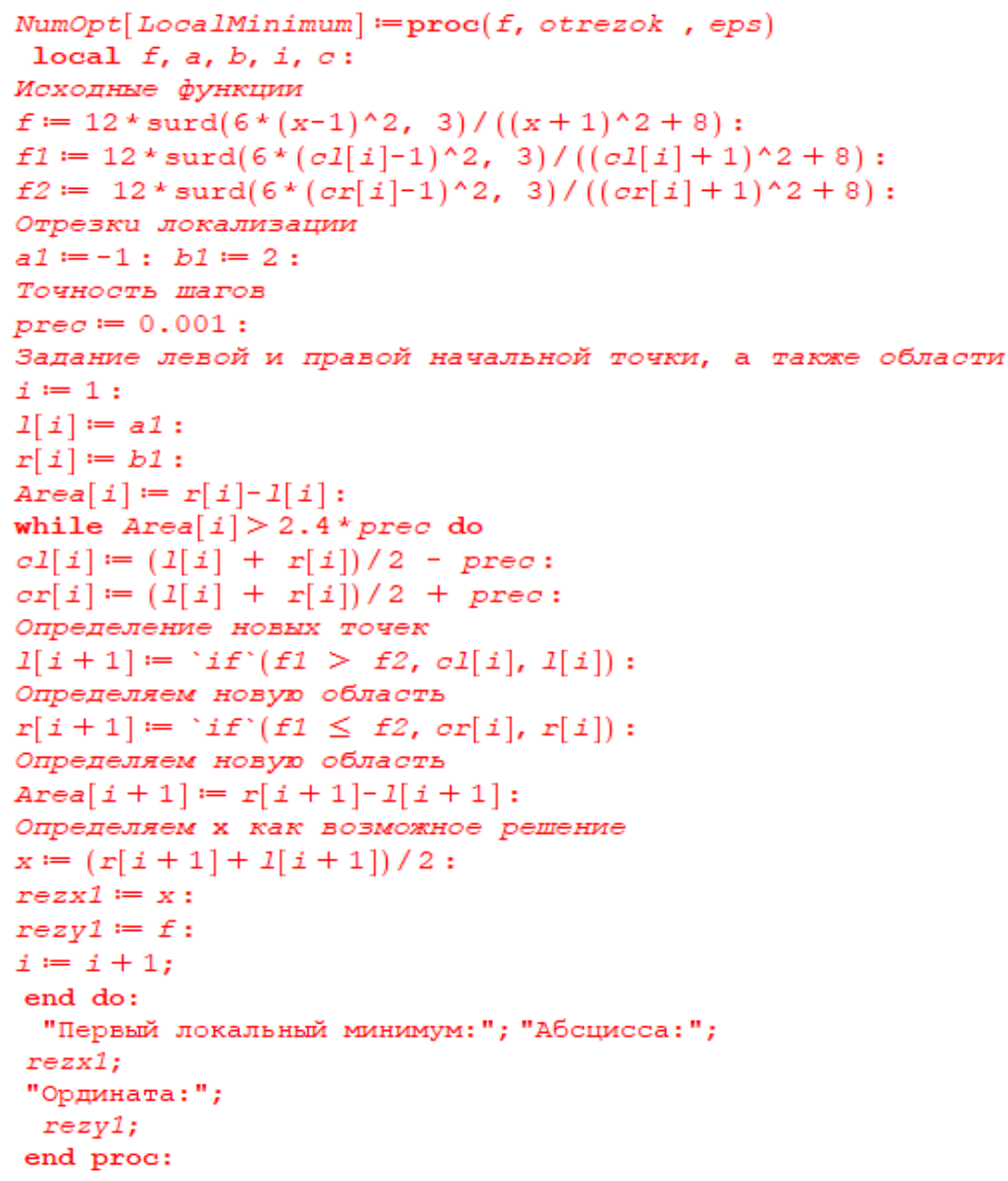

Library development

NumOpt_MuratM.MapleLib implements search of "Абсцисса:" ;

rezx1;

"Ордината: ";

rezy 1 ;

$i:=1$ :

$I[i]:=a 2:$

$r[i]:=b_{2}:$

Area $[i]:=r[i]-1[i]:$

Вынисление второго максимула

while Area $[i]>2.4 *$ prec do

$o l[i]:=(1[i]+r[i]) / 2$ - preo:

$\operatorname{ar}[i]:=(1[i]+r[i]) / 2+$ prec $:$

$I[i+1]:={ }^{\prime} i f(f 1<f 2$, ol $[i], I[i]):$

$r[i+1]:=f^{\prime}(f 1 \geq f 2$, or $[i], r[i])$ :

Area $[i+1]:=r[i+1]-1[i+1]:$

$\mathrm{x}:=(r[i+1]+1[i+1]) / 2:$

rezx $2:=x:$

rezy2 $:=f:$

$i:=i+1$;

end do:

"Второй локальный максимум" ;

"Абсцисса:" ;

rezx2;

"Ордината:";

rezy2;

"Глобальный максимум" ;

"Абсцисса:" ;

$\mathrm{xg} 1:=$ 'if'(rezy1> rezy2, rezx1, $r \in z \times 2): \mathrm{xg} 1$;

"ордината:"; local maxima and global maximum by the method of division in half 


\begin{tabular}{|c|c|c|c|c|c|c|}
\hline \multirow{4}{*}{ Impact Factor: } & ISRA (India) & $=3.117$ & SIS (USA) & $=0.912$ & ICV (Poland) & $=6.630$ \\
\hline & ISI (Dubai, UAI & $=0.829$ & РИНЦ (Russia) & $=0.156$ & PIF (India) & $=1.940$ \\
\hline & GIF (Australia) & $=0.564$ & ESJI (KZ) & $=8.716$ & IBI (India) & $=4.260$ \\
\hline & JIF & $=1.500$ & SJIF (Morocco) & $=5.667$ & OAJI (USA) & $=0.350$ \\
\hline
\end{tabular}

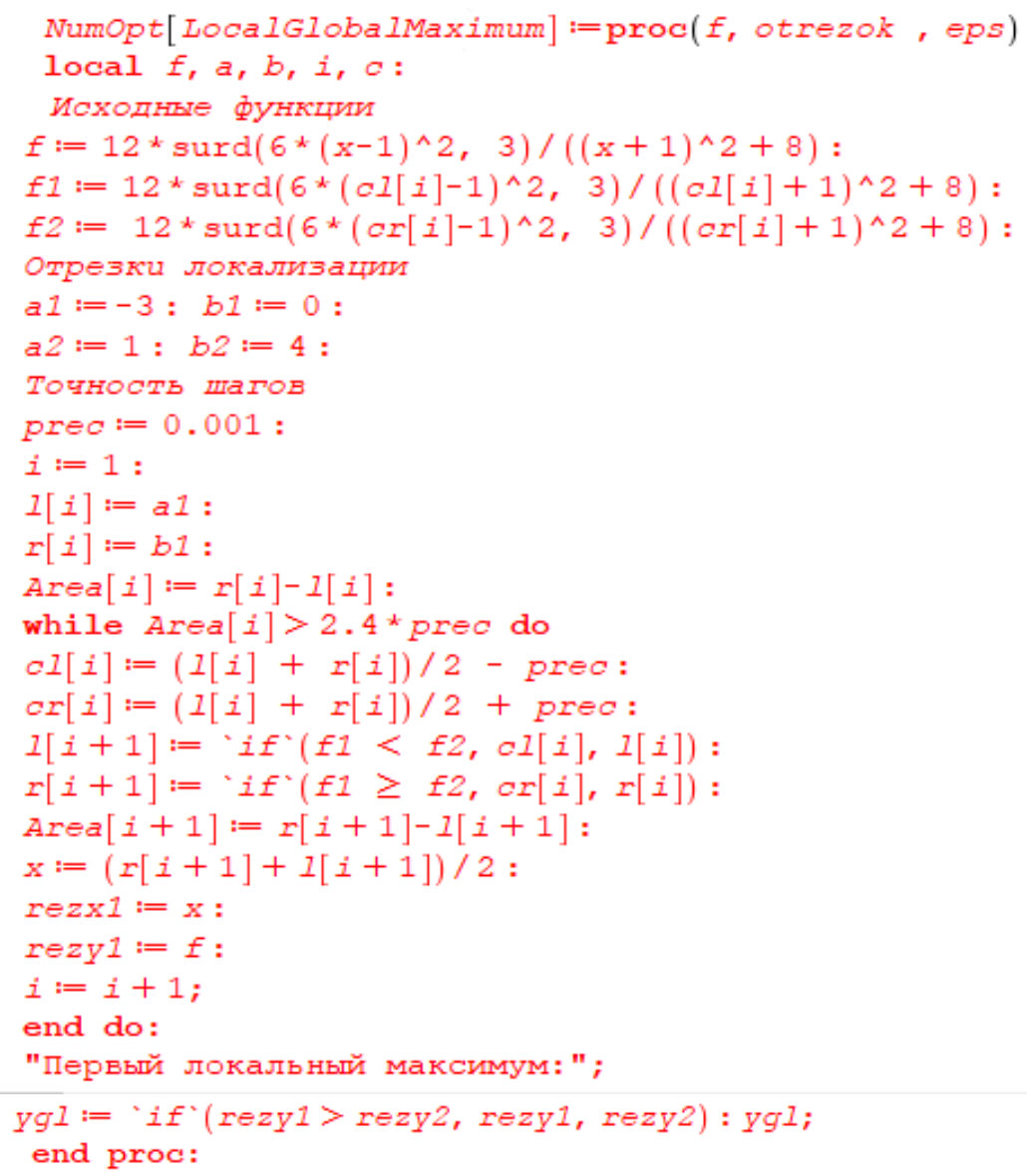

Library development NumOpt_MuratM.MapleLib implements the search

for the minimum function of two variables "by the method of the fastest descent»

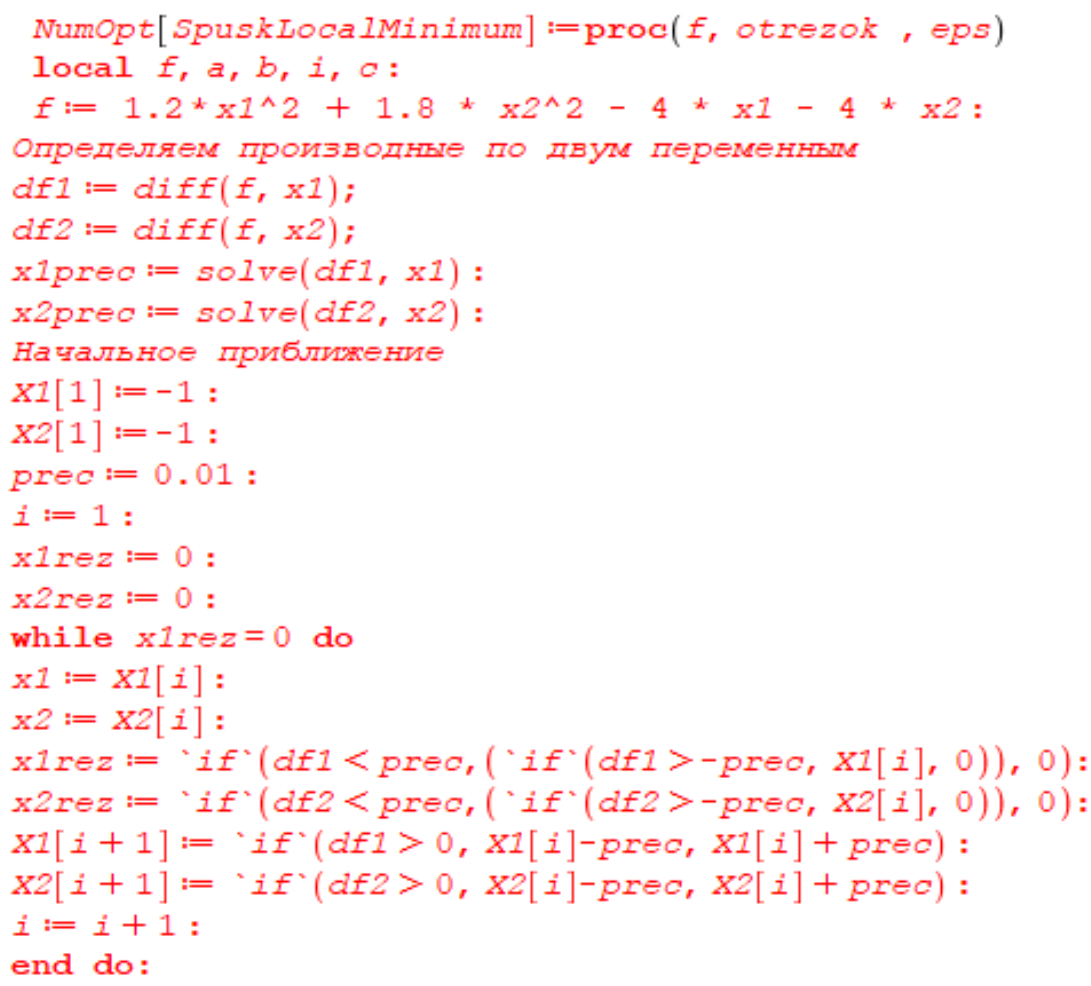




\begin{tabular}{|c|c|c|c|c|c|c|}
\hline \multirow{4}{*}{ Impact Factor: } & ISRA (India) & $=3.117$ & SIS (USA) & $=0.912$ & ICV (Poland) & $=6.630$ \\
\hline & ISI (Dubai, UAE & $=0.829$ & РИНЦ (Russia & $=0.156$ & PIF (India) & $=1.940$ \\
\hline & GIF (Australia) & $=0.564$ & ESJI (KZ) & $=8.716$ & IBI (India) & $=4.260$ \\
\hline & JIF & $=1.500$ & SJIF (Morocce & $=5.667$ & OAJI (USA) & $=0.350$ \\
\hline
\end{tabular}

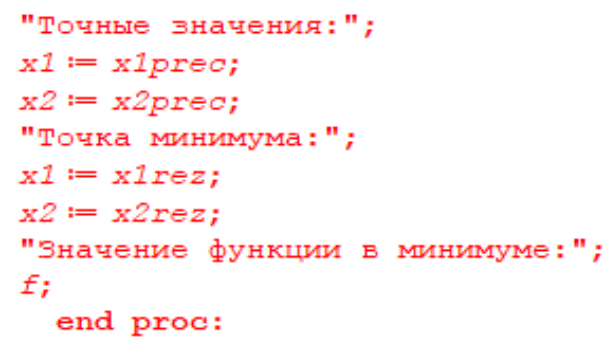

The considered methods give high accuracy of finding extremums of the function.

\begin{tabular}{|l|l|l|l|}
\hline & $\begin{array}{l}\text { Значения, найденные } \\
\text { средствами Марlе 7 }\end{array}$ & $\begin{array}{l}\text { Найденные методом } \\
\text { половинного деления }\end{array}$ & $\begin{array}{l}\text { Разница в результатах } \\
\text { Вычислений }\end{array}$ \\
\hline $\min$ & $(1,0)$ & $\begin{array}{l}(1.000093628, \\
0.003746629028)\end{array}$ & $\begin{array}{l}0.000093628, \\
0.003746629028\end{array}$ \\
\hline $1 \max$ & $(-2.0,5.039684200)$ & $\begin{array}{l}(-2.000093628, \\
5.039684194)\end{array}$ & $\begin{array}{l}0.000093628, \\
0.000000006\end{array}$ \\
\hline $2 \max$ & {$[\{x=3.0\}, 1.442249570]$} & $\begin{array}{l}(3.000093628, \\
1.442249569)\end{array}$ & $\begin{array}{l}0.000093628, \\
0.000000001\end{array}$ \\
\hline
\end{tabular}

\begin{tabular}{|c|c|}
\hline $\begin{array}{c}\text { Значения, найденные } \\
\text { методом наискорейшего } \\
\text { спуска }\end{array}$ & Значения, найденные средствами Maple 7 \\
\hline $\begin{array}{c}x I:=1.67 \quad x 2:=1.11 \\
y=-5,55554\end{array}$ & {$[\{x l=1.6666666667, x 2=1.111111111\},-5.5555555555]$} \\
\hline
\end{tabular}

\section{Conclusion}

As a result of the study, the algorithms of numerical methods for solving equations and solving numerical optimization problems based on the methods of half division, search for extrema functions, as well as the method of the steepest descent were studied. It can be concluded that:
- Algorithms on Maple for solving numerical optimization problems are obtained

- Numopt_muratm Library was developed.MapleLib implements numerical methods for solving equations and numerical optimization

- The library has been tested on several examples

- The resulting library can be used in research and calculations.

\section{References:}

1. Serikbaev, N., \& Shevtsov A. (2018). The development of a library of delphi for the solution of transcendental equations. ISJ Theoretical \& Applied Science, USA, 05 (61), 110. Doi: https://dx.doi.org/10.15863/TAS.2018.05.61.1

2. Zhunisbekov, S., Shevtsov, A., \& Karymsakov, N. (2018). Development of the simplest perceptron for artificial intelligence. ISJ Theoretical \& Applied Science, 06 (62), 1-10.
Soi: http://s-o-i.org/1.1/TAS-06-62-1 Doi: https://dx.doi.org/10.15863/TAS.2018.06.62.1

3. Kudryavtseva, I. V., Rykov, S., Rykov, S. V., \& Skobov, E. D. (2014). Optimization methods in the examples in the MathCAD 15 package. Part I:Studies'. manual. (p.166). SPb.: NRU ITMO, Aibt.

4. Shevtsov, A. N., et al. (2017). Innovative machines for post-harvest grain handling. ISJ Theoretical \& Applied Science, 01 (45), 171175. Soi: http://s-o-i.org/1.1/TAS-01-45-32 


\begin{tabular}{|c|c|c|c|c|c|c|}
\hline \multirow{4}{*}{ Impact Factor: } & ISRA (India) & $=3.117$ & SIS (USA) & $=0.912$ & ICV (Poland) & $=6.630$ \\
\hline & ISI (Dubai, UAE & $=0.829$ & РИНЦ (Russia) & $=0.156$ & PIF (India) & $=1.940$ \\
\hline & GIF (Australia) & $=0.564$ & ESJI (KZ) & $=8.716$ & IBI (India) & $=4.260$ \\
\hline & JIF & $=1.500$ & SJIF (Morocco) & $=5.667$ & OAJI (USA) & $=0.350$ \\
\hline
\end{tabular}

Doi:

https://dx.doi.org/10.15863/TAS.2017.01.45.32

5. Shevtsov, A., \& Talaybekkyzy, N. (2017).

Development of autonomous robot for radiation reconnaissance. ISJ Theoretical \& Applied Science, 03 (47), 1-4. Soi: http://s-oi.org/1.1/TAS-03-47-1 Doi: https://dx.doi.org/10.15863/TAS.2017.03.47.1

6. Abenov, U., \& Shevtsov, A. (2017). The application of system maple and delphi to solve problems of discrete mathematics. ISJ Theoretical \& Applied Science, 05 (49), 101119. Soi: http://s-o-i.org/1.1/TAS-05-49-17 Doi: https://dx.doi.org/10.15863/TAS.2017.05.49.17

7. Abenov, U., \& Shevtsov, A. (2017). The practical aspect of using greedy algorithm. ISJ Theoretical \& Applied Science, 05 (49), 120127. Soi: http://s-o-i.org/1.1/TAS-05-49-18 Doi:

https://dx.doi.org/10.15863/TAS.2017.05.49.18

8. Shevtsov, A., et al. (2017). Normalization of freshly harvested grain. ISJ Theoretical \& Applied Science, 05 (49), 249-256. Soi: http://so-i.org/1.1/TAS-05-49-40

Doi: https://dx.doi.org/10.15863/TAS.2017.05.49.40

9. Shevtsov, A. (2017). Development of an automatic dust collection system in mines. ISJ
Theoretical \& Applied Science, 08 (52): 1-4. Soi: http://s-o-i.org/1.1/TAS-08-52-1 Doi: https://dx.doi.org/10.15863/TAS.2017.08.52.1

10. Zhunisbekov, S., Shevtsov, A., \& Kairliyeva, D. (2017). On some aspects of the implementation of the principle of hamilton in maple. ISJ Theoretical \& Applied Science, 09 (53), 89-92. Soi: http://s-o-i.org/1.1/TAS-09-53-15 Doi: https://dx.doi.org/10.15863/TAS.2017.09.53.15

11. Koybakov, S., Maliktayuly, M., \& Shevtsov, A. (2017). Simulation models of chaotic flow. ISJ Theoretical \& Applied Science, 12 (56), 87-92. Soi: http://s-o-i.org/1.1/TAS-12-56-16 Doi: https://dx.doi.org/10.15863/TAS.2017.12.56.1

12. Serikbaev, N., \& Shevtsov, A. (2017) the development of a library of maple for the solution of transcendental equations. ISJ

Theoretical \& Applied Science, 12 (56), 261279. Soi: http://s-o-i.org/1.1/TAS-12-56-42 Doi:

https://dx.doi.org/10.15863/TAS.2017.12.56.42

13. Murat, M., \& Shevtsov, A. (2018). On some numerical optimization algorithms. ISJ Theoretical \& Applied Science, 12 (68), 1-5. Soi: http://s-o-i.org/1.1/TAS-12-68-1 Doi: https://dx.doi.org/10.15863/TAS.2018.12.68.1 